\title{
A METHOD BUILD FUZZY ASSOCIATE MEMORY FOR FUZZY CONTROL PROBLEMS
}

\author{
Ha Pham Thanh \\ Information Technology Section, University of Transport and Communications
}

Ho Nguyen Cat, Lan Vu Nhu

Institute of Information Technology, Vietnam Academy of Science and Technology

Received 31 May 2007

\begin{abstract}
Building fuzzy associate memory accordance with control problems has great meaning for control methods

In this paper we propose a method, that build fuzzy associate memory for control problem based on optimal trajectory of control problem, the method developed by theory hedge algebras and genetic algorithm
\end{abstract}

\section{INTRODUCTION}

In fact, many control problems have been solved by fuzzy approach, basically this approach uses a fuzzy associate memory being in form:

$$
\begin{aligned}
& \text { If } X_{1}=A_{11} \text { and } \ldots \text { and } X_{m}=A_{1 m} \text { then } Y=B_{1} \\
& \text { If } X_{1}=A_{21} \text { and } \ldots \text { and } X_{m}=A_{2 m} \text { then } Y=B_{2} \\
& \text { If } X_{1}=A_{n 1} \text { and } \ldots \text { and } X_{m}=A_{n m} \text { then } Y=B_{n}
\end{aligned}
$$

Where $A_{i j}$ and $B_{i}, i=1, \ldots, n, j=1, \ldots, m$, are verbal descriptions of physical variables $X_{j}$ and $Y$. With each value (or fuzzy value, or real value) of input variables, we have to define output value of $Y$.

According to the approach of fuzzy set theory, fuzzy control methods are based on the following idea: The meaning of linguistic values of linguistic variables in a fuzzy associate memory will be expressed by fuzzy sets. Since, each fuzzy associate memory will be imitated by a fuzzy dyadic relation $R$ and with an input vector $A_{0}$, the value of the output variable will be calculated by the formula: $B_{0}=A_{0} * R$, where $*$ is an aggregation operator.

With the generation and development of hedge algebra, a new fuzzy control method appeared which is called by a temporary name being Hedge Algebra used fuzzy control methods. This 
method is based on the idea: using quantified semantic mapping of hedge algebra, each fuzzy rule in a fuzzy associate memory will become a real point in $R^{n+1}$; with each input vector $A_{0}$ which has been quantified, the value of an output variable will be interpolated in a rule point set.

Normally, to construct a fuzzy associate memory they will refer experts' opinions in the domain, which the problem is examining, thus a fuzzy associate memory depends entirely on experts' experience. The experts' experience and the error model of the problem often are independent; therefore it's difficult for the control process to reach the accuracy. Desiring to overcome that problem, we propose a method to build fuzzy associate memory for fuzzy control problems; this method is based on the following idea:

Using hedge algebra to map a rule set into a real vector (rule vector).

Constructing a genetic algorithm to define a rule vector so that the error of the control is optimal, experts' experience is used to build genetic operators.

\section{HEDGE ALGEBRAS AND ALGORITHM OF CONTROL BASED ON HEDGE ALGEBRAS}

\subsection{Hedge algebras of linguistic variable (see $[8,9])$}

Given a linguistic variable $X$, denote by $\operatorname{Dom}(X)$ a set of linguistic terms of $X$ called a domain of $X$. For example, if $X$ is the rotation speed of a motor then $\operatorname{Dom}(X)=\{$ fast, very fast, more fast, little possibly fast, little fast, possibly fast, little slow, slow, possibly slow, very slow, more slow, very more slow,... $\} \cup\{0, W, 1\}$ is a domain of $X$. It can be considered as an algebra $A X=(\operatorname{Dom}(X), C, H, \leq)$, where $C$ is the set of generators which are the primary terms fast and slow, and the elements $W, O$ and 1 interpreted as the neutral, the least and greatest elements in $\operatorname{Dom}(X) ; H=\{$ very, little, possibly, more,... $\}$ is the set of hedges, which can be regarded as oneargument operations; the relation $" \leq "$ on $\operatorname{Dom}(X)$ is called a semantic ordering relation, because it is defined by the meaning of linguistic terms. The result of applying a hedge $h \in H$ to an element $x \in \operatorname{Dom}(X)$ is denoted by $h x$. For each $x \in \operatorname{Dom}(X)$, we denote by $H(x)$ the set of all elements $u \in \operatorname{Dom}(X)$ which are generated algebraically from $x$ by using hedges in $H$. That is $u$ can be expressed in the form $u=h_{0}, \ldots h_{1} x$, where $h_{1}, \ldots, h_{n} \in H$.

In the case the sets $H$ and $G$ are linearly ordered, $A X$ is called linear hedge algebra. For instance, the domain of the linguistic variable Truth: $T=\{$ true, false, very true, more true, possibly true, very false, possibly false, less false,... $\}$ can be considered as a linear hedge algebra $A T=(T, G$, $H, \leq$ ) with $G=\{$ true, false $\}, H=\{$ very, more, possibly, less (or little) $\}$ and $\leq$ is a relation induced by the meaning of terms in $X$, e.g. we have very true $>$ true, more true $>$ true, possibly true $<$ true, little true $<$ true, ...

Let us consider a hedge algebra $A X=(\operatorname{Dom}(X), C, H, \leq)$, where $H=H \cup H^{+}$, and suppose that $H^{-}=\left\{h_{-1}, h_{-2}, \ldots, h_{-q}\right\}$, where $h_{-1}<h_{-2}<\ldots, h_{-q}$, and $H^{+}=\left\{h_{1}, \ldots, h_{p}\right\}$, where $h_{l}<\ldots, h_{p}$ and $h_{0}=1$

Definition 2.1. An $\mathrm{fm}: X \rightarrow[0,1]$ is said to be a fuzziness measure (FM, for short) of terms in $X$ if:

$(f m l) f m(c)+f m\left(c^{+}\right)=1$ and for $\forall u \in X, \sum_{h \in H} f m(h u)=f m(u) ;$ In this case fm is called complete;

(fm2) If $H(x)=\{x\}$, then $f m(x)=0$. Especially, $f m(0)=f m(W)=f m(1)=0$; 
(fm3) $\forall x, y \in X, \quad \forall h \in H, \frac{f m(h x)}{f m(x)}=\frac{f m(h y)}{f m(y)}$, that is this proportion does not depend on specific elements and, hence, it is called the fuzziness measure of the hedge $h$ and denoted by $\mu(h)$.

The condition $f m l$ ) means that the primary terms and hedges under consideration is complete to model the semantics of real domains of a physical variable. $f m 2$ ) is quite natural $f m 3$ ) also seems to be rational: applying a hedge $h$ to different vague concepts, the relative modification effect of $h$ is the same!

It is easy to show the following,

Proposition 2.1. For fm and $\mu(h)$ defined in Def. 3.2, the following statements hold:

1) $f m(h x)=\mu(h) f m(x), \forall x \in X^{*}$;

2) $f m\left(c^{-}\right)+f m\left(c^{+}\right)=1$;

3) $\left.\sum_{-q \leq i \leq p, i \neq 0} f m\left(h_{i} c\right)\right\}=f m(c)$, where $c \in\left\{c^{-}, c^{+}\right\}$;

4) $\left.\sum_{-q \leq i \leq p, i \neq 0} f m\left(h_{i} x\right)\right\}=f m(x), x \in X$;

5) $\sum\left\{\mu\left(h_{i}\right):-q \leq i \leq-1\right\}=\alpha$ and $\sum\left\{\mu\left(h_{i}\right): 1 \leq i \leq p\right\}=\beta$, where $\alpha, \beta>0$ and $\alpha+\beta=1$.

Definition 2.2. (Sign function): Sign : $X \rightarrow\{-1,0,1\}$ is a function which is defined recursively as follows, where $h, h \in H, c \in\left\{c^{-}, c^{+}\right\}$:

a) $\operatorname{Sign}\left(c^{-}\right)=-1$, $\operatorname{Sign}\left(c^{+}\right)=+1$,

b) $\operatorname{Sign}\left(h^{\prime} h x\right)=0$, if $h^{\prime} h x=h x$ otherwise:

$\operatorname{Sign}\left(h^{\prime} h x\right)=-\operatorname{Sign}(h x)$, if $h^{\prime} h x \neq h x$ and $h^{\prime}$ is negative w.r.t. $h$ (or w.r.t. c, when $h=I$ and $x=c)$;

$\operatorname{Sign}\left(h^{\prime} h x\right)=+\operatorname{Sign}(h x)$, if $h^{\prime} h x \neq h x$ and $h^{\prime}$ is positive w.r.t. $h$ (or w.r.t. $c$, when $h=I$ and $x=c$ ).

Proposition 2.2. For $\forall x \in X, \forall h \in H$, if $\operatorname{Sign}(h x)=+1$ then $h x>x$, if $\operatorname{Sign}(h x)=-1$ then $h x<x$.

Definition 2.3. Let $\mathrm{AX}=(X, G, H, \leq)$ be a linear hedge algebra and $f m(c), f m\left(c^{+}\right)$and $\mu(h)$ be fuzziness measures of the primary terms $c^{-}, c^{+}$and hedge $h$, respectively, which satisfy 2) and 5) in Prop. 3.1. Then, $v$ is a mapping defined recursively as follows $(v$ is called a Quantified Semantic Mapping - SQM):

1) $v(W)=\theta=f m\left(c^{-}\right), v\left(c^{-}\right)=\theta-\alpha f m\left(c^{-}\right), v\left(c^{+}\right)=\theta+\alpha f m\left(c^{+}\right)$;

2) $v\left(h_{j} x\right)=v(x)+\operatorname{Sign}\left(h_{j} x\right)\left\{\sum_{i=s i g n(j)}^{j} f m\left(h_{i} x\right)-\omega\left(h_{j} x\right) f m\left(h_{j} x\right)\right\}$,

where $\omega\left(h_{j} x\right)=\frac{1}{2}\left[1+\operatorname{Sgn}\left(h_{j} x\right) \operatorname{Sgn}\left(h_{p} h_{j} x\right)(\beta-\alpha)\right] \in\{\alpha, \beta\}$, for all $j,-q \leq j \leq p$ and $j \neq 0$;

\subsection{Fuzzy Associative Memory (FAM) and Semantic Associative Memory (SAM)}

For a fuzzy controller, the collection of all its fuzzy rules is called the Fuzzy Associative 
Memory (FAM). Using SQM in definition 2.3, each of the fuzzy rules is a point in $\mathrm{R}^{\mathrm{m}+1}$ and this points is called the Semantic Associative Memory (SAM)

In fuzzy control, we often deal with multiple conditional fuzzy reasoning problems, whose the physical variables are normally modelled by linguistic variables, whose real domains usually are linearly ordered sets. So, hedge algebras as models of physical variable must be linear sets as well. This suggests us in this Section to deal with a new interpolation reasoning method to solve multiple conditional fuzzy reasoning problem and application to fuzzy control problems based on quantitative semantic mapping examined above.

Consider a fuzzy model (1)

Using fuzzy sets-based methods in fuzzy multiple conditional reasoning, we should carry out the following main steps:

1)To determine an appropriate reasoning method: One may choose a method based on composition rule (called also generalised Modus Ponens or fuzzy interpolation reasoning methods (see $[1,11,12]$ ). Note that their efficiency depends on a number of factors, such as implication operators, composition operators, aggregation operators and so on.

2)To determine fuzzy sets, i.e. membership functions: these functions should suitably represent the meaning of linguistic terms occurring in the fuzzy model and in the fuzzy input data, based on experts experiences and/or practical experiments.

3)To transform (in fuzzy control) the outputs of the method, which in general are also fuzzy sets, into real values by a defuzzification method.

It can be seen, in authors' opinion, the using these methods can be lose the intuition and meet with many difficulties to recognise their behaviours, since the results depend on several factors, whose influences on the chosen method can not be evaluated by mathematical methods.

Here we introduce a more intuitive approach, which based on interpolation reasoning methods. The idea is as follows to solve multiple conditional fuzzy reasoning problem with fuzzy model given by (1), we interpret each IF-THEN statement as defining a point and, therefore, this model defines a fuzzy curve $C_{j}$ in the Cartesian product $\operatorname{Dom}\left(X_{1}\right) \times \ldots \times \operatorname{Dom}\left(X_{m}\right) \times \operatorname{Dom}(Y)$, where $\operatorname{Dom}\left(X_{i}\right)$ and $\operatorname{Dom}(Y)$ are linguistic domains considered as hedge algebras of $X_{i}$ and $Y$, respectively. The, the fuzzy reasoning problem "For a given fuzzy model (4.1) and an input $A$ $\left(A_{1}, A_{2}, \ldots, A_{m}\right)$ find an output $B$ corresponding to $A$ " may be understood as an interpolation problem for the fuzzy curve $C_{j}$ in $\operatorname{Dom}\left(X_{1}\right) x \ldots x \operatorname{Dom}\left(X_{m}\right) \times \operatorname{Dom}(Y)$.

The main steps of our method are simply as follows:

1)To construct quantitative semantic mapping $v_{X i}$ and $v_{Y}$ which map the hedge algebras of $X_{i}$ and $Y$ into the unit interval $[0,1]$, respectively. As examined above, these mappings are determined by the fuzziness measure of primary terms and of linguistic hedges, which can be considered as users parameters to adapt specific applications.

2)Uder mappings $v_{X i}$ and $v_{Y}$ lunguistic values will be transformed into real values in $[0,1]$ and, hence, we can establish a transformation which transforms the fuzzy curve $C_{j}$ in $\operatorname{Dom}\left(X_{1}\right) x \ldots x \operatorname{Dom}\left(X_{m}\right) x \operatorname{Dom}(Y)$ into a real curve $\mathrm{C}_{r, m+1}$ in $\left[0, d_{l}\right] x\left[0, d_{2}\right] x \ldots x\left[0, d_{m}\right] x[0, b]$, where $\left[0, d_{l}\right]$ and $[0, b]$ are the domains of the basic variables of $X_{l}$ and $Y$, respectively.

3)Transform the real curve $C_{r, m+1}$ in Step 2) into a real curve $C_{r, 2}$ in $[0, a] x[0, b]$ by using an aggregation operator $\alpha$ (see [14] as follows :

$+a=\alpha\left(d_{1}, d_{2}, \ldots, d_{m}\right)$;

+ For each index $i, a_{i j}=v_{X i}(A i j)$ for $j=1, \ldots, m$, we determine a point $\left(a_{i}, b_{i}\right)$ of $C_{r, 2}$ by the following equations: 
$a=\alpha\left(d_{i 1}, d_{i 2}, \ldots, d_{i m}\right) ;$ and $b_{i}=v_{Y}\left(B_{i}\right)$;

Use the classical linear interpolative method to compute the output corresponding to an input data $a_{0}=\alpha\left(v_{X 1}\left(A_{01}\right), v_{X 2}\left(A_{02}\right), \ldots, v_{X m}\left(A_{0 m}\right)\right)$, for the given input terms $X_{1}=A_{01}, \quad X_{2}=A_{02}, \ldots, X_{m}$ $=A_{0 m}$.

\subsection{Algorithm of control based on hedge Algebras}

Sections 2,3,4 describe all elements and operation needed in a Hedge Algebras-based Controller (HAC). Figure 1. shows the general schema of HAC.

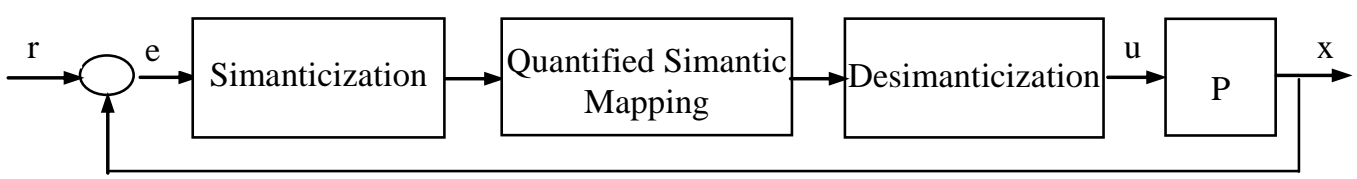

Fig. 1: Hedge Algebras-based Controller.

where $r$ is reference, $e$ is error and $u$ is control action, $P$ is plant.

For purposes of simplicity, we consider the simple two-input one output case in order to emphasize and clearify the basis ideas of the proposed approach.

Let a set of desired input-output data pairs is given from FAM

$$
(e, \Delta e ; u)
$$

where $e$ and $\Delta e$ are inputs and $u$ is the output of HAC; $\Delta e=\dot{e}$.

Step 1: Simanticization maps the universe of discourse $\left[\mathrm{y}^{-}, \mathrm{y}^{+}\right]$of a linguistic variable onto a simantic operating range [s0,s1] with linear form of scaling:

$$
y_{s}=\frac{(s 1-s O)\left(y-y^{-}\right)}{y^{+}-y^{-}}+s O
$$

For example: generate quantified semantic intervals based on domain input, output intervals. Assume that the domain intervals of $e, \Delta e$ and $u$ are

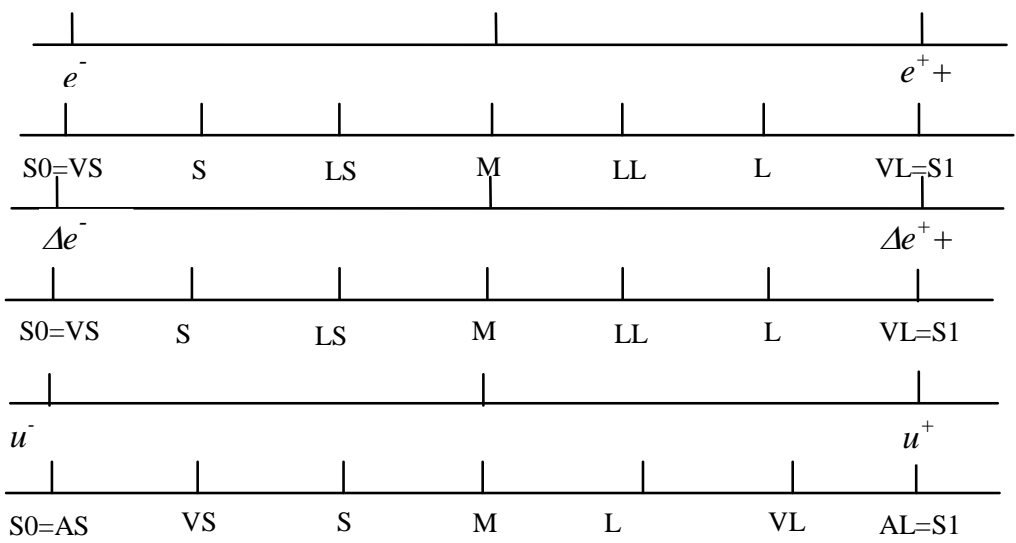

Fig. 2: The interval construction of QSM on the given domain intervals of e, $\Delta e$ and $u . M$ : Medium; S: Small; LS: Little Small; VS: Very Small; AS: Absolute Small; L: Large; LL: Little Large; VL: Very Large; AL: Absolute Large. 


$$
\left[e^{-}, e^{+}\right],\left[\Delta e^{-}, \Delta e^{+}\right] \text {and }\left[u^{-}, u^{+}\right], \quad \text { respectively }
$$

where the domain interval of a variable means that most probably this variable will lie in this interval. Construct quantified semantic interval equivalently to each domain interval of the inputs, output variables as follow:

Step 2 :

Determine quantified semantic mapping as follows : The semantic rules determined in this way, are "AND" rules, i.e rules in which the condition of the IF input semantic part may be PRODUCT of the semantic input points or MIN of the semantic input points in order for results of the THEN of the semantic output part to occur. The results are collected in table 1 .

Table 1: (for example) 49 Semantic rules, respectively with 49 semantic output points of quantified semantic mapping. It is Semantic Associative Memory (SAM).

\begin{tabular}{|c|c|c|c|c|c|c|c|}
\hline $\mathrm{x}_{1}$ & VS & $\mathrm{L}$ & LL & M & LS & $S$ & VS \\
\hline VL & AL & VL & $\mathrm{L}$ & $\mathrm{L}$ & L & $\mathrm{L}$ & $\mathrm{M}$ \\
\hline $\mathrm{L}$ & VL & VL & L & $\mathrm{L}$ & L & $\mathrm{M}$ & $S$ \\
\hline LL & $\mathrm{L}$ & $\mathrm{L}$ & $\mathrm{L}$ & $\mathrm{L}$ & $\mathrm{M}$ & $S$ & $\mathrm{~S}$ \\
\hline M & $\mathrm{L}$ & $\mathrm{L}$ & $\mathrm{L}$ & M & $S$ & $S$ & $S$ \\
\hline LS & $\mathrm{L}$ & $\mathrm{L}$ & $\mathrm{M}$ & $S$ & $S$ & $\mathrm{~S}$ & $S$ \\
\hline$S$ & $\mathrm{~L}$ & $\mathrm{M}$ & $S$ & $S$ & $S$ & VS & VS \\
\hline VS & $\mathrm{M}$ & $S$ & $S$ & $S$ & $S$ & VS & AS \\
\hline
\end{tabular}

Define the interpolative quantified semantic mapping (curve) based on SAM.

Step 3: Desimanticization maps the semantic range of the control action [s1, s0] onto the operating range of the control action $\left[\mathrm{u}^{-}, \mathrm{u}^{+}\right]$:

A linear form of scaling is given by

$$
u=\frac{\left(u^{+}-u^{-}\right)\left(u_{s}-s O\right)}{s 1-s O}+u^{-}
$$

For given real numerical data inputs $\left(e^{0}, \Delta e^{0}\right)$, the quantified semantic curve is used to determine the output semantic control $u_{s}$ and the corresponding element of the output real control $u$.

From Step 1 to Step 3 one can see that the proposed algorithm is more simple than conventional algorithm of fuzzy control in the sence that does not require defuzzification strategy. Its design requires choice of:

+ the number of membership functions

+ the shape of membership functions

+ the definition of fuzzy implication

a measure of central tendency ( center of mass ) of the membership functions;

Hence, Hedge Algebras approach has some advantages over the fuzzy approach, namely, it is simple, quick to construct, more intuitive and more exactly. 


\section{GENETIC ALGORITHM (GA)}

\subsection{An overview}

A GA carries out a process of searching the optimal answer according to many ways, by maintaining a population of solutions, and fostering the exchange of information between these solutions. The population experiences an evolutionary process: each generation will reproduce fairly good solutions, and fairly bad solutions will die. To distinguish between different solutions, an objective function is used to play a part as an environment.

A structure of a simple genetic algorithm is similar to a structure of any evolutionary algorithm. In each step, like $t$, genetic algorithm maintain a population of solutions (chromosomes, vectors), $P(t)=\left\{x_{1}^{t}, \ldots, x_{n}^{t}\right\}$. Each solution $x_{i}^{t}$ is valued to specify its adaptability. A new population will be generated by keeping best-ranking individuals. A number of individuals of this population experience changes by crossover and mutation, and create new solutions. Breeding combines characteristics of parents' chromosomes to create child's chromosomes by permuting correlative sections of genes of parents; breeding allows exchanging information between solutions. Unlike crossover, mutation modifies accidentally one or more genes of the selected chromosome with a probability showing mutating rate, mutation allow adding new information into the population, thus it makes hereditary factors more abundant $[6,12]$.

\subsection{The classical genetic algorithm}

Procedure GA()

$\{$ //Solving optimization problems

$\mathrm{k}=0$;

//Initiating randomly a population $P_{0}$.

//Evaluating the value of the objective functions of each individual.

Initialize $\left(\mathrm{P}_{\mathrm{k}}\right)$;

Evaluate_the_objective_function $\left(\mathrm{P}_{\mathrm{k}}\right)$;

//Assigning the individual having the best value of the objective function

I/to the solution of the algorithm

$\mathrm{X}_{\text {best }}=\operatorname{Best}\left(\mathrm{P}_{\mathrm{k}}\right)$;

Do \{

//Changing the value of the objective function into the fitness and

//selecting and breeding the parent population

$\mathrm{P}_{\text {parent }}=\operatorname{Select}\left(\mathrm{P}_{\mathrm{k}}\right)$;

$/ / B r e e d i n g$ and mutating to create the child population

$\mathrm{P}_{\text {Child }}=\operatorname{Mutation}\left(\operatorname{Breed}\left(\mathrm{P}_{\text {parent }}\right)\right)$;

//Replacing the current population with the child population

$\mathrm{k}=\mathrm{k}+1$

$\mathrm{P}_{\mathrm{k}}=\mathrm{P}_{\text {child; }}$;

Evaluate_the_objective_function $\left(\mathrm{P}_{\mathrm{k}}\right)$; 
IIIf the value of the objective function of the best-ranking individual X

// in the population $P_{k}$ is greater than the value of the objective function

// of $X_{\text {best }}$, the solution will be replaced

$$
\mathrm{X}=\operatorname{Best}\left(\mathrm{P}_{\mathrm{k}}\right) \text {; }
$$

If $\left(\right.$ obj $(X)>$ obj $\left.\left(X_{\text {best }}\right)\right) X_{\text {best }}=X$;

\}while ( $<\mathrm{G})$; //Performing $\mathrm{G}$ generations

Return $\left(\mathrm{X}_{\text {best }}\right)$; / returning the solution of the $G A$

\}

There are 6 respects needing to be considered when you apply the genetic algorithm to solve a problem:

+ Encoding solutions into a string of individuals.

+ The fitness function.

+ The diagram of selecting parent individuals.

+ The crossover operator.

+ The mutation operator.

+ Replacing strategy or reproduce operator.

There are many solutions to these problems, for example the canonical genetic algorithm Holland uses binary encoding for solution individuals. The objective function (the function need optimizing) is selected to be the basis of measuring the fitness of each individual string. Then the fitness of each individual is used to calculate selecting probability. The used selecting diagram is a selecting diagram by rate, in this diagram an individual having the fitness $f_{i}$ has selecting probability being $p_{i}=\frac{f_{i}}{\sum_{j=1}^{N} f_{j}}$, where $\mathrm{N}$ is the quantity of individuals of the population. Crossover operator is a one-point cut operator; suppose the length of the individual string is $L$ (the individual string has $L$ bits), breeding is carried out through two phases:

+ Selecting randomly two individuals in the parent population following the discrete uniform distribution.

+ Generating randomly a number $\mathrm{j}$ in $[1, L-1]$. Two child individuals are created by coping characters from $l$ to $j$, and changing the positions of characters from $j+l$ to $L$

\section{Two parent individuals \\ 1001110101 \\ 0100111110 \\ Position of breeding \\ Two child individuals \\ 1001110110 \\ 0100111101}

The mutation operator is constructed as follow: examining each gene of the child individual which has been produced after performing the crossover operator and changing 0 into 1 or 1 into 0 with a probability $p_{m}$ known as mutating probability.

Replacing strategy or reproduce operator will be performed last. The child population, which was produced from the parent population by using three operators as: selection, crossover and mutation, will totally replace the current population of next generation. 
A genetic algorithm depends on a 4- tuple, $\left(N, p_{c}, p_{m}, G\right)$, where $\mathrm{N}$ - the number of individuals of a population; $\mathrm{p}_{\mathrm{c}}$ - breeding population, $\mathrm{p}_{\mathrm{m}}$ - mutating population, and $\mathrm{G}$ - the number of generations that need to be evolved are controlling parameters of the algorithm. An individual, which has the value of the objective function being best through all generations, is the final solution of algorithm, the initial population is chosen randomly $[6,12,13]$

\subsection{Improvements of the canonical genetic algorithm}

The canonical genetic algorithm meets converging difficulties; to overcome them they have been proposed many improving methods. In the main, improving doesn't change the structure of the algorithm but concentrate on encoding solutions, for example, instead of encoding a solution into a binary string, we can encode the solution into a real vector, hence crossover and mutation operators are built more flexibly in order to bring the genetic algorithm to converge to the global minimum (maximum) value, for example, the mutation operator is built to a group of synchronous mutating, unsynchronous mutating, the crossover operator is also built to a group of simple breeding and arithmetical breeding [4].

\section{A METHOD TO BUILD A FUZZY ASSOCIATIVE MEMORY}

\subsection{A method}

As presenting in section 1, the fuzzy controlling method using hedge algebra uses a semantic quantified mapping (SQM) to change a fuzzy associative memory (FAM) into a semantic associative memory (SAM), hence each fuzzy rule in the fuzzy associative memory is expressed as a real point in $m+1$ - dimensional space $R^{m+1}$, therefore a fuzzy associative memory consisting of $m$ rules can be expressed into a vector having $(m+1) n$ real elements.

The problem is finding a vector so that the error of the control is acceptable, and obviously the problem is altered into a problem of optimizing an error function of control, and we propose using the genetic algorithm to find this vector.

The generated fuzzy associative memory have to harmonize two factors: being appropriate to experts' experience, and ensuring to approach optimal trajectory of control problems. Thus we propose using experts' experience to build genetic operators.

Through deploying the following application, we demonstrate the practicability of this solution.

\subsection{Aircraft landing control problem (see[13])}

The dynamic equation:

$$
\begin{aligned}
& h(i+1)=h(i)+v(i) \\
& v(i+1)=v(i)+f(i)
\end{aligned}
$$

Where $v(i), h(i), f(i)$ is in turn the velocity, height and controlling force of aircraft at the moment $i$.

For this problem we assume that the universe of discourse for the three variables are as follows: $0 \mathrm{ft} \leq \mathrm{h}(\mathrm{i}) \leq 1000 \mathrm{ft} ;-30 \mathrm{ft} / \mathrm{s} \leq \mathrm{v}(\mathrm{i}) \leq 30 \mathrm{ft} / \mathrm{s} ;-30 \mathrm{lbs} \leq \mathrm{f}(\mathrm{i}) \leq 30 \mathrm{lbs}$ and $h(0)=1000 \mathrm{ft} ; \mathrm{v}(0)=20 \mathrm{ft} / \mathrm{s}$

The optimal trajectory of the aircraft landing has the following form:

$$
v=-\left(-20 /(1000)^{2}\right) / h^{2}(\text { see }[7,8])
$$

Errors of landing velocity $\left(e_{F}\right)$ through $\mathrm{n}$ controlling cycles are defined by 


$$
e_{F}=\left(\sum_{i=1}^{n}\left(v_{i 0}(F)-V_{i}(F)\right)^{2}\right)^{1 / 2}(\text { see }[7,8])
$$

Where: $v_{i 0}(F)$ is the optimal landing velocity at cycle i corresponding $h(i), v_{i}(F)$ is the landing velocity at cycle i corresponding $h(i)$.

Experts' experience shows that:

- When the aircraft is in "high", if velocity is rising, controlling force will be controlled to go down.

- When the aircraft is in "low", if velocity is going down, controlling force will be controlled to rise.

Controlling force to go down makes the velocity reduce bit by bit following height.

The problem is constructing a rule set for this control problem so that the error of control is minimum, on the other hand the rule set must be suitable to pilots' experience.

\subsection{Building fuzzy associative memory}

\subsubsection{Constructing the hedge algebras for linguistic variable of problem}

In this section we construct a hedge algebra for both 3 linguistic variable $\mathrm{v}, \mathrm{h}$ and $\mathrm{f}$ follow as:

$\mathrm{C}=\{$ Small, Medium, Large $\}$

$\mathrm{H}=\{$ Litle, Very $\}$

$\theta=\alpha=\beta=0.5$

Consider we only use fuzzy sets of linguistic value of hedge algebra with depth $\mathrm{d}=2$. We have: $\mathrm{H}($ Small $)=\{$ "VerySmall","Small","LitleSmall" $\}$

H(Large $)=\{$ "LitleLarge","Large","VeryLarge" $\}$

$\mathrm{H}(\mathrm{G})=\mathrm{H}(\mathrm{Smal}) \cup\{$ Medium $\} \cup \mathrm{H}($ Large $)=\{$ "VerySmall", "Small", "LitleSmall", "Medium", "LitleLarge", "Large", "VeryLarge"\}

Using SQM for $v, h$ and $f$ we have

$v(\mathrm{H}(\mathrm{Smal}))=\{0.125,0.25,0.375\}$

$v(\mathrm{H}($ Large $))=\{0.625,0.75,0.875\}$

$v($ Medium $)=0.5$

$v(\mathrm{H}(\mathrm{G}))=\{0.125,0.25,0.375,0.5,0.625,0.75,0.875\}$

In the fact, we can using label fuzzy sets as: \{DownLarge, DownMedium,DownSmall, Zero, UpSmall, UpMedium,UpLarge \} instead of fuzzy sets in $\mathrm{H}(\mathrm{G})$

The interval construction of QSM on the given domain intervals $\mathrm{h}, \mathrm{v}$ and $\mathrm{f}$ defined by functions $h T O h s(h), v T O v s(v), f T O f s(f)$ and $h s T O h(h s), v s T O v(v s), f s T O f(f s)$ are opposite function of $h \operatorname{TOhs}(h), v \operatorname{TOvs}(v)$ and fTOfs $(f)$ with $h^{-}=0, h^{+}=1000, h s^{-}=0, h s^{+}=1 ; v^{-}=-30, v^{+}=30, v s^{-}=0, v s^{+}=1$; $f=-30, f^{+}=30, f s^{-}=0, f s^{+}=1$ ( see step 1,3 section 3.2)

Using aggregation operator AND $=$ PRODUCT when constructing the quantified semantic curve and we have SAM $\left.=\left\{\left(h s(i) * v s(i), f_{s}(i)\right), \mathrm{i}=1 . . \mathrm{n}\right\}\right)$ and conclusion control can determine by a linear interpolation function Interpolation $\left(h s^{*} v s, S A M\right)$ 


\subsubsection{Constructing the genetic algorithm}

A genetic algorithm is used to solve the optimization problem, in this problem we will use a genetic algorithm to define a fuzzy associative memory so that the error of controlling process is optimal, and the algorithm is:

\section{Encoding solutions}

A solution (SAM table) is encoded into a real vector $\left(a_{1}, \ldots, a_{n m}\right)$ with conditions:

$-a_{i} \in v(H(G)), \mathrm{i}=1, \ldots, \mathrm{nm}$, where $a_{j}, \ldots, a_{j+m-l}$ is the rule $\mathrm{j}, \mathrm{j}=1, \ldots, \mathrm{n}$

- The rule $\mathrm{j}$ must be suitable to pilots' experience;

\section{Choosing an initial population}

The initial population including $\mathrm{P}$ solutions is choosing randomly so that each rule of a solution is appropriate to pilots' experience.

The object function (the error function) is built as follow:

The error at each controlling cycle $k(k=0, \ldots, n)$ is defined by:

+ Real height and velocity:

If $k=0$ then $h(k)=1000$

Else $h(k)=h(k-1)+v(k-1)$;

If $k=0$ then $v(k)=-20$

Else $v(k)=v(k-1)+f(k-1)$;

+ Correlative height and velocity following hedge space defining mapping:

$h s(k)=h T O h s(h(k))$;

$v s(k)=v \operatorname{TOv} s(v(k))$;

+ Correlative force following height and velocity

$f(k)=\operatorname{Interpolation}(h s(k) * v s(k), S A M)$

+ Correlative force following the inverse mapping of hedge space defining mapping:

$f(k)=f s T O f\left(f_{s}(k)\right)$;

+ Optimal velocity: $v o(k)=-\left(20 / 1000^{2}\right) /(h(k))^{2}$

+ Error at each cycle: $e(k)=(v o(k)-v(k))^{2}$

The objective function is sum of errors of $n$ cycles: $g(S A M)=\sum_{k=1}^{n} s(k)$

The function $g(S A M)$ is used to be basis to measure the fitness of solution individuals

Using selecting diagram following rate of Holland (see section 3.2)

\section{Constructing crossover and mutation operators}

Due to the condition of the problem $a_{i} \in v(H(G))$ crossover and mutation operators have to construct so that solutions after crossover and mutation will have genes being in the condition set $v(H(G))$. On the other hand, the result of crossover and mutation has to ensure that each rule in $S A M$ is appropriate to pilots' experience, thus we use these experience to building crossover and mutation operators. 
+Crossover operator: We still use one point-cut crossover operator; however breeding only is performed if the point-cut is the gene corresponding with height of a rule of the solution. The crossover like this guarantees that the reasonableness of the rule set isn't broken by pilots' experience.

+Mutation operators: Mutating in a solution only concentrates on genes k corresponding the controlling force $(\mathrm{k} \bmod 3=0)$, when we carry out mutating, we examine genes $\mathrm{k}-2$, $\mathrm{k}-1$ corresponding height and velocity of rule k-2, pilots' experience are used as follow (see (2))

+ if the value of gene $k-2 \geq v$ (Medium) //The aircraft is in "high"

then if the value of gene $k-1 \geq v$ (Medium) //The velocity is rising

then replacing the value of gene $\mathrm{k}$ with a random value $<v$ (Medium)

//Reducing controlling force

+ if the value of gene $\mathrm{k}-2<v($ Medium) //The aircraft is in "low"

then if the value of gene $k-1<v$ (Medium) //The velocity is going up

then replacing the value of gene $k$ with a random value $\geq v$ (Medium)

\section{//Increasing controlling force}

Breeding and mutating following a breeding probability $P c$ and a mutating probability $P m$.

\subsubsection{Results}

With the genetic algorithm constructed above, the number of generations is $G=2000$, the breeding probability is 0.8 , and mutating probability is 0.03 , the number of rules, that need to be found, is $M=15$, the size of the population is $\mathrm{P}=30$, the objective function is calculated through $\mathrm{n}=50$ controlling cycles; through some tests, we found the following rule set:

Table 2: The new rule set of the aircraft control.

\begin{tabular}{cccc}
\hline No & Height $(\boldsymbol{h})$ & Velocity $(\boldsymbol{v})$ & Controlling force $(\boldsymbol{f})$ \\
\hline 1 & Medium & Zero & DnMedium \\
2 & LitleLarge & Zero & DnMedium \\
3 & Medium & DnLarge & Zero \\
4 & Large & DnLarge & DnSmall \\
5 & Large & DnLarge & DnSmall \\
6 & LitleSmall & DnLarge & Zero \\
7 & VerySmall & Zero & DnSmall \\
8 & Small & UpSmall & DnSmall \\
9 & VerySmall & DnSmall & UpSmall \\
10 & LitleSmall & DnSmall & Zero \\
11 & Medium & DnSmall & Zero \\
12 & Small & DnSmall & UpSmall \\
13 & Small & DnSmall & UpSmall \\
14 & Medium & UpMedium & DnMedium \\
15 & Large & DnSmall & DnSmall \\
\hline
\end{tabular}


The controlling result of using the rule set through 4 controlling cycles is calculated and shown by figure 2 :

Table 3: The controlling result.

\begin{tabular}{|c|c|c|c|c|c|c|c|c|c|}
\hline \multicolumn{7}{|c|}{ Hedge Algebras-based Controller for $A N D=P R O D U C T$} & \multirow{2}{*}{\multicolumn{3}{|c|}{$\begin{array}{c}\text { Fuzzy Conventional } \\
\text { Controller[13] }\end{array}$}} \\
\hline \multicolumn{4}{|c|}{ Using new rule set } & \multicolumn{3}{|c|}{ Using old rule set [7] } & & & \\
\hline$k$ & $h(k)$ & $v(k)$ & $f(k)$ & $h(k)$ & $v(k)$ & $f(k)$ & $h(k)$ & $v(k)$ & $f(k)$ \\
\hline 1 & 1000.0 & -20.00 & 0.28 & 1000 & -20 & 0 & 1000 & -20 & 5.8 \\
\hline 2 & 980.0 & -19.72 & 0.12 & 980 & -20 & 1 & 980 & -14.2 & 0.5 \\
\hline 3 & 960.3 & -19.61 & 0.32 & 960 & -19 & 7.5 & 965.8 & -14.7 & -0.4 \\
\hline 4 & 940.7 & -19.29 & 0.11 & 941 & -11.5 & -13.5 & 951.1 & -15.1 & 0.3 \\
\hline
\end{tabular}

Total velocity errors after 4 control cycles of $\mathrm{HAC}$ and FC can be defined as :

$$
\begin{aligned}
& \mathrm{e}_{\mathrm{F}}=\left(\sum_{\mathrm{i}=1}{ }^{4}\left(v_{\mathrm{i} 0}(F)-v_{\mathrm{i}}(F)\right)^{2}\right)^{1 / 2}=7.15 \\
& \mathrm{e}_{O-H A P}=\left(\sum_{\mathrm{i}=1}^{4}\left(v_{i 0}(O-H A P)-v_{i}(O-H A P)\right)^{2}\right)^{1 / 2}=6.12 \\
& e_{O-H A P}=\left(\sum_{i=1}{ }^{4}\left(v_{i O}(N-H A P)-v_{i}(N-H A P)\right)^{2}\right)^{1 / 2}=2.04
\end{aligned}
$$

$e_{F}$ is total downward velocity error of $\mathrm{FC}$ by the method

$e_{O-H A P}$ is total downward velocity error of HAC with old rule set for AND=PRODUCT

$e_{N-H A P}$ is total downward velocity error of HAC with new rule set for AND=PRODUCT

$v_{i 0}(F)$ is desired downward velocity at i-th cycle with the same height of FC

$v_{i 0}(O-H A P)$ is desired downward velocity at i-th cycle with the same height of HAC in the case AND=PRODUCT and using old rule set

$v_{i 0}(N-H A P)$ is desired downward velocity at i-th cycle with the same height of HAC in the case AND=PRODUCT and using new rule set

$v_{\mathrm{i}}(\mathrm{F})$ is downward velocity at $\mathrm{i}$-th cycle of $\mathrm{FC}$ by the method

$v_{i}(O-H A P)$ is downward velocity at $\mathrm{i}$-th cycle of $\mathrm{HAC}$ with old rule set for AND=PRODUCT

$v_{i}(N-H A P)$ is downward velocity at $\mathrm{i}$-th cycle of $\mathrm{HAC}$ with old rule set for AND=PRODUCT

Through comparing the control result with the canonical rule set of the aircraft landing control problem and the former with the rule set that we built (see table 3 and equations 4.4, 4.5, 4.6), we realize that the controlling error with this new rule set is much better than the error with the old rule set $(2.04<<<6.12<7.15)$

\section{CONCLUSION}

The article has been proposed a new solution to building fuzzy associative memories for fuzzy control problems, ensuring that the error of controlling process is optimized and the rule set is appropriate to experts' experience. 


\section{REFERENCES}

1. Cao, Z. and Kandel, A. (1989), Applicability of some fuzzy implication operators, Fuzzy sets and systems 31, pp. 151-186.

2. Davis, L. Ed., (1987), Genetic algorithms and simulated annealing, Pitman Publishers, London, England.

3. Goldberg, D.E. (1989), Genetic algorithm in search, optimization and machine learning, Addison-Wesley, Reading, Massachusets.

4. Holland, J.H. (1975), Adaptation in natural and artificial systems. The University of Michigan Press.

5. Ho, N.C. (2003), Quantifying Hedge Algebras and Interpolation Methods in Approximate Reasoning, Proc. of the 5th Inter. Conf. on Fuzzy Information Processing, Beijing, March 1-4, pp. 105-112.

6. Ho, N.C., Lan, V.N., and Viet, L.X. (2006), Quantifying Hedge Algebras. Interpolative reasoning method and its application to some problems of fuzzy control WSEAS TRANSACTIONS ON COMPUTER. Issue 11, Vol. 5, pp. 2519- 2529.

7. Ho, N.C., Lan, V.N., Viet, L.X. (2006), An interpolative reasoning method based on Hedge Algebras and its application to a problem of fuzzy control", Proceedings of the 10th WSEAS International on COMPUTERS, Vouliagmeni, Athens, Greece, July 13-15, pp. 526-534.

8. Cat-Ho Nguyen (2007), A topological completion of refined hedge algebras and a model of fuzziness of linguistic terms and hedges. Fuzzy Sets and Systems 158(4), pp. 436-451.

9. Cat-Ho Nguyen and Nguyen Van Long (2007), Fuzziness measure on complete hedge algebras and quantifying semantics of terms in linear hedge algebras. Fuzzy Sets and Systems 158(4), pp. 452-471.

10. Koza, J.R. (1990), Genetic Programming: A Paradigm for Genetically Breeding Populations of Computer Programs to Solve Problems, Report No.STAN-CS-90-1314, Stanford University.

11. Kiszka, J.B, Kochanska, M.E., and Sliwinska, S. The influence of some fuzzy implication operators on the accuracy of a fuzzy model-Part I, Fuzzy Sets and Systems 15(1983), 111 128.

12. Kiszka, J.B, Kochanska, M.E., and Sliwinska, S. The influence of some fuzzy implication operators on the accuracy of a fuzzy model-Part II, Fuzzy Sets and Systems 15(1983),223240.

13. Ross, T.J. Fuzzy logic with Engineering application. International Edition. Mc Graw-Hill, Inc 2004 (second Ed).

14. Yager, R.R (1994), Aggregation operators and fuzzy systems modelling. Fuzzy Sets and Systems, 67, pp. 129-145. 